\title{
Histochemische und polarisationsoptische Untersuchungen an ultraviolett-bestrahlter Haut
}

\author{
Von W. Hanke \\ Aus dem Zoologischen Institut der Johann-Wolfgang-Goethe-Universität Frankfurt a. M. \\ (Z. Naturforschg. 12 b, 299—302 [1957]; eingegangen am 18. Oktober 1956)
}

\begin{abstract}
Untersuchungen an uv-bestrahlten Schwimmhäuten von Fröschen und Rückenhäuten von Mäusen ergaben, daß mit Hilfe der Nadi-Reaktion (GRÄFF) Oxydationspotentiale an der Bestrahlungsstelle nachgewiesen werden können. Kurzwelliges UV unter $\lambda=300 \mathrm{~m} \mu$ hat hierbei die beste Wirkung. Die Stellen stärkster Oxydationskraft liegen bei Schwimmhäuten an den Melanophoren des Corium, bei Mäuserücken-Häuten verteilt im Corium. Bei den Froschhäuten kann zusätzlich bei unphysiologischem $p_{\mathrm{H}} 4,2$ Oxydationswirkung in der Epidermis nachgewiesen werden. Strukturelle Veränderungen an der Schwimmhaut werden polarisationsoptisch deutlich.
\end{abstract}

Die Untersuchung der Haut nach Bestrahlung mit ultraviolettem Licht hat bisher zur Auffindung verschiedener Wirkungsprinzipien geführt. Einige Autoren richteten ihr Augenmerk auf histamin-ähnliche Stoffe und andere pharmakologisch aktive Agenzien. Ebenso standen die Redoxeigenschaften bestimmter Hautschichten nach UV-Anwendung im Vordergrund. So konnten Wels, Urban und Mitarbb. ${ }^{12,14,15}$ feststellen, daß die Reduktionswirkung des Stratum germinativum nach UV-Bestrahlung zunimmt. Andere Autoren (Dubouloz, Dumas und Mitarbb. ${ }^{2,3}$ ) fanden eine Bildung fettlöslicher Peroxyde in der Haut nach Einwirkung verschiedener Agenzien, darunter UV, Wärme und Röntgenstrahlen.

In vitro ist das Eintreten von Oxydationen durch Bestrahlung mit sichtbaren und UV-Strahlen allgemein bekannt (s. u. a. Schenck ${ }^{11}$ ). Die Reaktionsweise von Röntgenstrahlen und anderen ionisierenden Strahlen stellt man sich heute allgemein so vor, daß vor allem eine Bildung von aktiviertem Wasser erfolgt. Bei Gegenwart von molekularem Sauerstoff soll es dabei leicht zu Wasserstoffperoxyd-Bildung (LANGendorfF ${ }^{9}$, HorNykiewytsch ${ }^{8}$ ) kommen. Ähnlich könnte man sich, falls die Energie ausreicht, die Entstehung von Oxydationsprodukten nach Bestrahlung mit UV vorstellen.

Die eigenen Untersuchungen gingen von Beobachtungen an den Kapillaren der Frosch-Schwimmhaut aus (Giersberg und Hanke ${ }^{5}$ ). Der Blutstrom in den Kapillaren zeigt nach Bestrahlung mit UV Stase im

1 U. Böhmer u. H. Giersberg, Naturwissenschaften 43, 303 [1956].

2 P. Dubouloz u. J. Dumas, Proc. of the first International Photobiological Congress, Amsterdam 1954, S. 247.

3 P. Dubouloz, J. Dumas u. J. Vigne, C. R. Séances Soc. biol. Filiales 144, 1080 [1950]. bestrahlten Bereich. Es handelt sich um eine Erscheinung, bei der anscheinend zunächst kein Fibringerüst ausgebildet wird, um eine echte Stase also (nach WEBER ${ }^{13}$ „Konglomeration“). Die Arteriolen verändern ihre Weite nicht, wodurch eine Auslösung dieses Effektes durch Histamin oder Adrenalin ausscheidet. Die Feststellung von chemischen Veränderungen erfolgte im Anschluß an den Stasenvorgang an der Schwimmhaut dieser Kaltblüter. Bisher konnte nur das Verschwinden einer histamin-ähnlichen Substanz, die aber keinesfalls mit Histamin identisch ist, als radiochemischer Prozeß in der Froschhaut nachgewiesen werden (Böhmer und Giersberg ${ }^{1}$ ).

\section{Methodik}

Als Strahlenquelle diente ein Quarzquecksilber-Hochdruckbrenner von ca. $80 \mathrm{~W}$ (Hg-Br.) der Firma Osram sowie ein Quecksilber-Niederdruckbrenner (Nd-Br.) der Hanauer Höhensonnengesellschaft. Der $\mathrm{Hg}$-Br. wurde teilweise mit den Filtern WG 7 und WG 6 kombiniert.

Grundsätzlich wurde eine Quarzküvette (Küv. 1, 2 oder 3) mit fließendem Wasser (Schichtdicke ca. $10 \mathrm{~mm}$ ) zwischengeschaltet, so daß keine Verbrennung durch langwellige Wärmestrahlen erfolgen konnte. Bestrahlt wurde ein kreisrunder Bezirk von 2,5 mm Durchmesser der feuchtgehaltenen Schwimmhaut. Der Frosch (Rana temporaria L.) war mit Urethan betäubt.

Die energetische Ausmessung der auf diese kleine Fläche auffallenden Strahlung ist schwierig, und so erlauben die angegebenen Werte nur eine Orientierung über die Intensitäten der einzelnen Linien. Nach vielen Variationen erfolgte die Bestrahlung schließlich unter den in der Tab. 1 angegebenen Standardbedingungen.

${ }^{4}$ F. Duspiva, Enzymatische Histochemie in: Hoppe-SeylerThierfelder, Hdb. der physiologisch. und pathol.-chem. Analyse.

5 H. Giersberg u. W. Hanke, Z. vergleich. Physiol. 37, 128 [1955].

6 D. Glick, Techniques of Histo- and Cytochemistry, New York 1949. 


\begin{tabular}{|c|c|c|c|c|}
\hline & $\begin{array}{c}\text { Nd-Br. } \\
\text { Abst. 4,5 cm } \\
\text { Küv. 1 } \\
\text { o. Filter }\end{array}$ & $\begin{array}{c}\text { Hg-Br. } \\
\text { Abst. 4,5 cm } \\
\text { Küv. 2 } \\
\text { o. Filter }\end{array}$ & $\begin{array}{c}\mathrm{Hg}-\mathrm{Br} . \\
\text { Abst. 4,5 cm } \\
\text { Küv. 2 } \\
\text { WG 7 }\end{array}$ & $\begin{array}{c}\text { Hg-Br. } \\
\text { Abst. 4,5 cm } \\
\text { Küv. 2 } \\
\text { WG6 } 6\end{array}$ \\
\hline & & & & \\
254 & 100 & 76 & - & - \\
266 & - & 265 & 15 & - \\
276 & - & 105 & 15 & 5 \\
280 & - & 130 & 25 & 5 \\
290 & - & 170 & 70 & 30 \\
297 & ca 2 & 430 & 260 & 150 \\
303 & - & 620 & 400 & 260 \\
313 & ca 1 & 1050 & 820 & 670 \\
334 & - & 255 & 230 & 220 \\
366 & ca 2 & 3400 & 3200 & 3050 \\
\hline
\end{tabular}

Tab. 1. Bestrahlungsstärken der wichtigsten Wellenlängen im UV (Genauigkeit etwa 10\%). Wellenlänge in $\mathrm{m} \mu$; Bestrahlungsstärke in $\mu \mathrm{W} / \mathrm{cm}^{2}$.

Nach Beendigung der Bestrahlung wurden Gefrierschnitte quer durch ein Gebiet hergestellt, das zum Vergleich bestrahlte und unbestrahlte Regionen enthielt. Der Nachweis erfolgte mit N a d i - Reagenz ( $\alpha$-Naphthol und Dimethyl-p-phenyldiamin nach GRÄFF ${ }^{7}$, RomeIs ${ }^{10}$, GLICK $^{6}$ ). Es ist dies ein Reagenz auf Cytochromoxydase, das aber auch auf andere oxydierende Substanzen anspricht. Bei Organen mit starkem Stoffwechsel erfolgt der Farbniederschlag an den Mitochondrien schon nach einigen Minuten. Die Querschnitte durch die FroschSchwimmhäute mußten mehrere Stdn. in der Lösung verbleiben, um einen eindeutigen Farbausfall zu erzielen. Verschiedenes $p_{\mathrm{H}}$ bei der Anwendung des $\mathrm{N}$ a di Reagenzes hatte unterschiedliche Erfolge.

\section{Ergebnisse der histochemischen Untersuchungen}

Die normale Schwimmhaut stellt für die histologische Untersuchung ein sehr übersichtliches Objekt dar. Die Epidermis umrahmt beiderseits das Corium, das mit geschlängelt verlaufenden Zellelementen das Innere ausfüllt. In der Mitte liegt noch eine dünne Tela subcutanea, die einige Hohlräume, die Lymphräume, einschließt und die sich durch den Faserverlauf nur schwer vom Corium unterscheiden läßt. Das Stratum germinativum besteht aus senkrecht zur Oberfläche stehenden länglichen Zellen mit großen Kernen mit lockerem Chromatingerüst. Nach außen schließen sich mehrere Zellagen mit abgerundeten Kernen an, deren äußerste Zellen allmählich absterben; die Kerne werden pyknotisch. Diese Zellen gehen in das Stratum corneum über, dessen Kerne

7 S. GRÄFF, Zbl. allg. Pathol. pathol. Anatom. 32, 337 [1922].

8 Th. Horny niewytsch, Strahlentherapie 86, 175 [1952].

9 H. Langendorff, Strahlentherapie 85, 391 [1951].

10 B. Romeis, Mikroskopische Technik, München 1948.

11 G. O. Schenck, Z. Naturforschg. 3 b, 59 [1948].

* Abb. $1-6$ s. Tafel S. 302 a u. b. durch Hämatoxylin und ähnliche Kernfarbstoffe nicht mehr charakteristisch angefärbt werden (Abb. $1^{*}$ ).

Bei histochemischer Untersuchung der normalen Schwimmhaut mit $\mathrm{Nadi-Reagenz} \mathrm{zum} \mathrm{Nachweis}$ der Cytochromoxydase bei $p_{\mathrm{H}} 7,0-7,4$ erscheint nach längerer Einwirkung die Epidermis blau. Es treten viele blaugefärbte winzige Granulen auf, die Orte starker Oxydationskraft (Mitochondrien) in der Zelle bezeichnen. Im Corium treten die Granulen weniger auf. Bei $p_{\mathrm{H}} 4,2-4,4$ läßt sich diese Erscheinung nicht bemerken; sowohl Epidermis als auch Corium erscheinen ungefärbt. Die Reaktion ist also in diesem Fall an physiologisches $p_{\mathrm{H}}$ gebunden, jedoch spricht die längere Einwirkungszeit des Reagenzes dafür, daß die Cytochrom-Oxydase und ihre Reaktionsprodukte in dieser stoffwechselträgen Kaltblüterhaut mengenmäßig sehr gering sind. Die Reaktionsprodukte dieser Oxydase benötigen lange Anreicherungszeit, um deutlich sichtbares Indophenolblau auszufällen. Die Mitochondrien dienen als Niederschlagszentren. Nach Moog (zit. n. Duspiva ${ }^{4}$ ) hemmt Natriumazid die Cytochrom-Oxydase. Bei den hier beobachteten Reaktionen ist Natriumazid allerdings ohne Bedeutung für den Reaktionsausfall. Allerdings konnte auch in zur Kontrolle angefertigten Mäuseleber-Querschnitten das angewandte $\mathrm{Na}$ triumazid die $\mathrm{Nadi}$ - Reaktion nicht unterdrücken, so daß seine Wirkung auf die Cytochrom-Oxydase in meinen Versuchen fraglich ist. Im Corium treten in geringem Umfang in der unbestrahlten Schwimmhaut bei $p_{\mathrm{H}} 7,2$ dicke Niederschläge von Indophenolblau auf, die an Melanophoren oder Blutgefäßen liegen. Diese erscheinen sehr vereinzelt und sind nicht so gleichmäßig verteilt wie die granulöse Blaufärbung von Epidermis und Corium.

Histologisch lassen sich nach Bestrahlung mit dem $\mathrm{Hg}$-Br. (gesamtes UV) nach 120 Min. in der Epidermis strukturelle Auflösungen feststellen. Die feste Begrenzung nach außen durch das Stratum corneum löst sich auf. Die Kerne der Epidermis werden zunächst vakuolisiert und schließlich pyknotisch (Abb. 2). Für diese Erscheinung sind die verschiedenen beteiligten Wellenlängen in unterschiedlichem Umfang verantwortlich. Die Durchdringungs-Fähigkeit der wirksamen Strahlung ist so groß, daß auch in

\footnotetext{
12 G. Urban, Proc. of the first International Photobiological Congress, Amsterdam 1954, S. 260.

${ }_{13}$ H. W. WeBer, Klin. Wschr. 33, 387 [1955].

14 P. Wels, Naunyn-Schmiedebergs Arch. exp. Pathol. Pharmakol. 208, 116 [1949].

${ }^{15}$ P. Wels u. Mitarbb., Forsch. u. Fortschr. 28, 257 [1954].
} 
der abgewandten Epidermis Vakuolisierung der Kerne erfolgt. Die Anteilnahme der Wellenlängen an der Vielfalt der histologischen Veränderungen ist durch Filter nicht zu erschließen. Hierfür wurden monochromatische Bestrahlungen (gemeinsam mit H. B ü cker, Max-Planck-Institut für Biophysik, Frankfurt a. M.) vorgenommen, die auch Aufschluß über die Eindringtiefe gestatten. Hierüber wird an anderer Stelle berichtet.

Mit Nadi-Reagenz zeigt sich eine Anhäufung der dicken Indophenolblau-Niederschläge im Corium der Bestrahlungsstelle bei $p_{\mathrm{H}}$ 7,2. Diese Reaktion tritt verstärkt an den Melanophoren und Blutgefäßen der abgewandten Seite auf. Dies beruht darauf, daß die Melanophoren der zugewandten Seite von wesentlich mehr Energie getroffen werden und dadurch bereits häufig in Auflösung begriffen sind. In den Blutgefäßen setzt nach dieser Bestrahlung Stase ein. Die Zerstörung der Blutkörperchen in den Kapillaren der zugewandten Seite ist stärker. Die Epidermis auf der Seite des Strahleneinfalles ist blauer als die der abgewandten Seite gefärbt. Ebenso nimmt das Corium eine diffuse Blaufärbung an. Bei $p_{\mathrm{H}} 4,2$ ist die Blaufärbung der bestrahlten Epidermis noch wesentlich deutlicher, weil die unbestrahlte Epidermis dagegen farblos erscheint. Niederschläge von Indophenolblau an Melanophoren und Blutgefäßen des Coriums treten nicht auf (Abb. 3 und 4 ).

Mit $\mathrm{N}$ a di - Reagenz sind also zwei verschiedene Oxydationsreaktionen des bestrahlten Gewebes nachzuweisen. Erstens stellen bei $p_{\mathrm{H}} 7,2$ die noch nicht zerstörten Melanophoren und wohl in geringerem Umfang auch die Blutgefäße Ausgangsorte erhöhter Oxydationskraft dar, die einen dicken Niederschlag von Indophenolblau zur Folge haben. Dies ist eine physiologische Reaktion, die in wesentlich geringerem Umfang auch im unbestrahlten Bezirk vorliegen kann. Zweitens erhöht sich das Oxydationspotential in der Epidermis auf der Strahlen-Einfallsseite außerordentlich und geringer im Corium. Der hierdurch bewirkte Ausfall von Indophenolblau ist ziemlich diffus verteilt und beruht auf Reaktionen, die auch noch im Bereich von $p_{\mathrm{H}} 4,0-4,4$ ablaufen. Im stark sauren Bereich allerdings hört auch dieser Effekt auf, wahrscheinlich wird aber hier die Indophenolblau-Reaktion an sich gestört.

Bei Bestrahlung mit dem Nd-Br. treten erst nach 240 Min. eindeutig nachweisbare Reaktionen auf. Nach 120 und 180 Min. Bestrahlung sind die Erfolge unklar und schwankend. Deutlich ist nach 240 Min. Bestrahlung die Verstärkung des IndophenolblauNiederschlages an den Melanophoren des Corium an der Bestrahlungsstelle bei $p_{\mathrm{H}}$ 7,2 zu erkennen. Diese Reaktion ist mindestens ebenso eindeutig nachzuweisen wie bei Bestrahlung mit dem $\mathrm{Hg}$-Br. Dagegen ist der Nachweis der diffusen Epidermisund Coriumbläuung nur sehr ungenau. Wenn vorhanden, so ist die Bläuung sehr schwach und undeutlich. Wahrscheinlich ist hierzu eine wesentlich höhere Leuchtdichte im bestrahlten Gebiet notwendig, die aber mit dem Nd-Br. noch nicht zu erreichen ist. Strukturelle Veränderungen der Epidermis lassen sich histologisch nicht nachweisen, doch werden die Kerne schon vakuolisiert und teils pyknotisch.

Bei Nd-Br.-Bestrahlung tritt im allgemeinen ebenfalls Stase ein, wenn auch in einzelnen Fällen nichts - trotz verdoppelter zeitlicher Einwirkung gegenüber Hg-Br. - zu bemerken ist. Prinzipiell ist jedenfalls die Wellenlänge $\lambda=254 \mathrm{~m} \mu$ zur Auslösung der Stase befähigt. Die spektrale Wirkungskurve wird bei der Diskussion monochromatischer Bestrahlung erörtert werden.

Bei Bestrahlung mit Hg-Br. und Filter WG 7 sind vor allem die Wellenlängen des UV C $(<280 \mathrm{~m} \mu)$ stark gedämpft. Bei Versuchen mit verschieden langen Bestrahlungszeiten waren die $\mathrm{Nadi}$-Reaktionen zwar erzielbar, aber nicht in allen Fällen vorhanden. Bei 135 - 190 Min. Bestrahlungszeit treten die Indophenolblau-Niederschläge etwas geschwächt auf. Die diffuse Bläuung, die auch im unphysiologischen $p_{\mathrm{H}} 4,2$ nachzuweisen ist, ist nur schwach vorhanden, scheint also höhere Energie der geschwächten Wellenlängen zu benötigen.

Bei Bestrahlung mit dem Filter WG 6 treten nach einer Bestrahlungszeit von 240 Min. die ersten meist noch undeutlichen Ergebnisse auf. Vor allem verstärkte Bläuung in der bestrahlten Epidermis ist beobachtbar. Im Gegensatz zur Bestrahlung mit ungefiltertem UV erscheinen die Niederschläge an den Melanophoren nur unklar.

Aus den Filterversuchen kann mit einiger Sicherheit geschlossen werden, daß die Wellenlängen, die für diese beschriebenen Effekte verantwortlich sind, im Bereich des UV C und UV B liegen. Die Wirksamkeit sinkt nach $300 \mathrm{~m} \mu \mathrm{ab}$. Das Maximum der Wirkung liegt wohl bei $280 \mathrm{~m} \mu$ und $297 \mathrm{~m} \mu$. Jedoch läßt sich durch diese Versuche nicht eindeutig klären, ob $\lambda=254 \mathrm{~m} \mu$ allein geringere Wirkung hat, ob also 
die Wirkkurve zu den kurzen Wellenlängen hin wieder absinkt.

Nach diesen an der übersichtlichen FroschSchwimmhaut gewonnenen Ergebnissen sollte auch an der Rückenhaut von weißen Mäusen nach oxydierenden Stoffen geforscht werden. Hierzu wurde eine Partie der Rückenhaut enthaart, die Mäuse mit Pernocton betäubt und bestrahlt. Direkt nach der Bestrahlung mit dem vollen UV des $\mathrm{Hg}$-Br. entnommene Häute zeigten keine verstärkte Bläuung durch Indophenolblau im bestrahlten Bereich. Dagegen konnte beobachtet werden, daß schon nach 1-2 Stdn. im bestrahlten Hautgebiet, das während dieser Zeit normal an der Maus verblieb, eine starke Bildung von Indophenolblau auftritt, verteilt vor allem im Corium bis zum subcutanen Fettgewebe hin. Dies geschieht nur bei $p_{\mathrm{H}} 7,2$. Nach etwa $10 \mathrm{Stdn}$. ist die Indophenolblau-Bildung sehr stark. Die Verteilung geht von einzelnen Punkten aus, die strukturell noch nicht näher identifiziert sind. Die Reaktion ist den Niederschlägen an den Melanophoren vergleichbar. Allerdings sind die Konzentrationspunkte nicht so groß wie die Niederschläge an den Melanophoren, weil die Absorption der Energie an diesen ja besonders hoch ist (Abb. 5). Die Ansammlung von Oxydations-Elementen findet sich in der Rückenhaut nach Bestrahlung von 90 Min. mit dem $\mathrm{Hg}-\mathrm{Br}$. wenige Stdn. nach der Bestrahlung.

\section{Ergebnisse der Untersuchung von Strukturveränderungen}

Zur Veränderung der Hautstruktur nach Bestrahlung sollen hier zunächst nur einige Bemerkungen gemacht werden. Polarisationsoptisch ergibt sich an den unbestrahlten Froschhaut-Querschnitten folgendes Bild: Der Faserverlauf im Stratum corneum ist parallel zur Oberfläche; senkrecht dazu stehen die Zellen des Stratum germinativum, die nur geringe Aufhellung des Polarisationslichtes zeigen. Mitten im Corium liegen in geringem Umfang parallel zur Oberfläche ausgerichtete Fasern. Im übrigen verlaufen die Coriumfasern kreuz und quer. Nach der intensiven Bestrahlung mit dem vollen $\mathrm{UV}$ des $\mathrm{Hg}$-Br. läßt sich die Auflösung der Epidermis daran erkennen, daß das Stratum corneum an der bestrahlten Seite die Aufhellung durch den parallelen Faserverlauf nicht mehr zeigt. Dagegen haben sich im Corium dicke Bündel parallel zur Oberfläche liegender Fasern gebildet, die je nach Dauer der Bestrahlung verschieden weit das Innere der Schwimm. haut ausfüllen. Diese Erscheinung läßt sich vielleicht durch Wasserverlust, der enges Zusammenrücken der Strukturelemente zur Folge hat, erklären. Meistens ist die Haut an der bestrahlten Stelle dünner (Abb. 6).

Die polarisationsoptischen Untersuchungen erfolgten an Gefrierschnitten in $p_{\mathrm{H}} 4,1-5,0$ (Phenolwasser). An fixierten Schnitten zeigte sich infolge Denaturierung eine stärkere Aufhellung bei gekreuzten Polarisations-Filtern im Corium, so daß die Struktur-Veränderungen nicht mehr deutlich werden.

\section{Diskussion der Ergebnisse}

Über die Natur der nachgewiesenen OxydationsPotentiale läßt sich zunächst noch nichts Näheres aussagen. Während bei der Maus nur im neutralen $p_{\mathrm{H}}$ eine erhöhte Indophenolblau-Abscheidung im bestrahlten Bereich festzustellen war, zeigte sich beim Frosch in der Epidermis und im Corium auch bei $p_{\mathrm{H}}$ 4,0 eine Oxydations-Reaktion. Diese Oxydationskraft läßt sich nur schwer mit Oxydasen erklären, weil fast keine $p_{\mathrm{H}}$-Abhängigkeit vorliegt. Dubouloz und Dumas ${ }^{2,3}$ haben Peroxyde nachgewiesen, die sich durch Aceton ausziehen lassen. Einlegen der Haut oder der Schnitte in Aceton führte bei den bisherigen Versuchen zu keiner Veränderung im Reaktionsausfall, sowohl die diffuse Indophenolblau-Verteilung als auch die Niederschläge an den Melanophoren lassen sich durch Aceton nicht ausziehen. Natriumazid, das die Cytochrom-Oxydase hemmen soll, hat ebenfalls keine Verschiedenheit herbeigeführt. Nach Fixierung des Gewebes in Formol-Alkohol fehlen alle Reaktionen, es treten höchstens sehr schwache Epidermis-Bläuungen auf. Die Oxydationseffekte lassen sich also nur am unfixierten Gewebe nachweisen. Hervorzuheben ist die verstärkte Oxydationskraft an den Melanophoren; meistens expandieren dieselben im bestrahlten Bezirk. Die Melanophoren auf der zugewandten Seite sind bei Bestrahlung mit der vollen Energie des $\mathrm{Hg}$-Br. oft zerstört, teils aber Ausgangspunkte des Indophenolblau-Niederschlages. Von hoher Bedeutung ist die Tatsache, $\mathrm{da}$ in der Mäusehaut die Reaktionen erst nach mehreren Stdn. auftreten. Ein Vergleich mit der Bildung des Erythems liegt nahe, das ja ebenfalls eine Latenzzeit besitzt.

Weitere Versuche zur Klärung der Natur der oxydierenden Substanzen sind im Gange.

Der Firma Osram danke ich, daß sie mir Höchstdruckbrenner vom Typ HBO 2001 zur Verfügung stellte. 


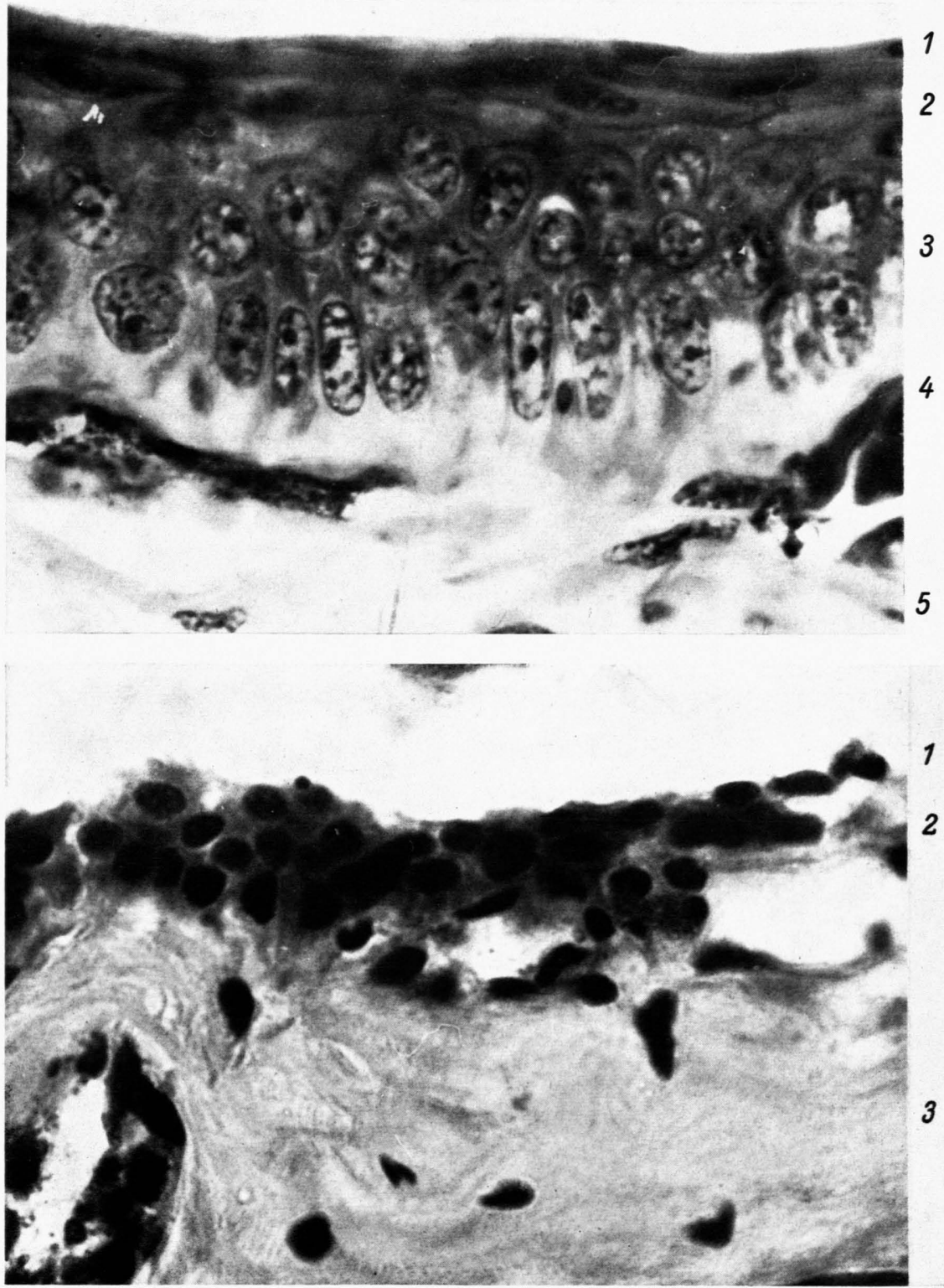

1

2

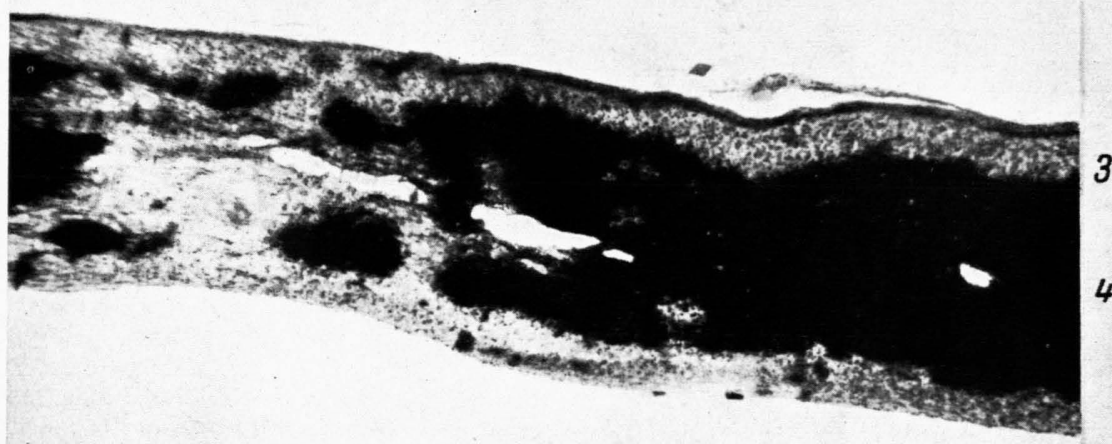

Abb. 1. Unbestrahlte Epidermis (Vergr.ca.1600-fach). 1 Stratum corneum, 2 Übergangsschicht, 3 Stratum germinativum, 4 Lage der Melanophoren und kleinen Gefäße, 5 Corium.
Abb. 2. Bestrahlte Epidermis (Vergr. ca. 1500-fach). Bestrahlt mit Hg-Br/120 Min.; 1 aufgelöstes Str. corneum, 2 Str. germinativum mit pyknotischen Kernen, 3 Corium.

Abb. 3. Einwirkung von $\mathrm{N}$ a d i Reagenz auf die bestrahlte Schwimmhaut bei $p_{\mathrm{H}} 7,2$ (Vergr. ca. 130-fach). Bestrahlt mit NdBr. 240 Min. 1 unbestr. Stelle, 2 bestrahlte Stelle, 3 Epidermis, 4 Corium mit dickem Indophenolblau-Niederschlag an der bestrahlten Stelle.

Bereitgestellt von | Universitaetsbibliothek Frankfurt/Main 


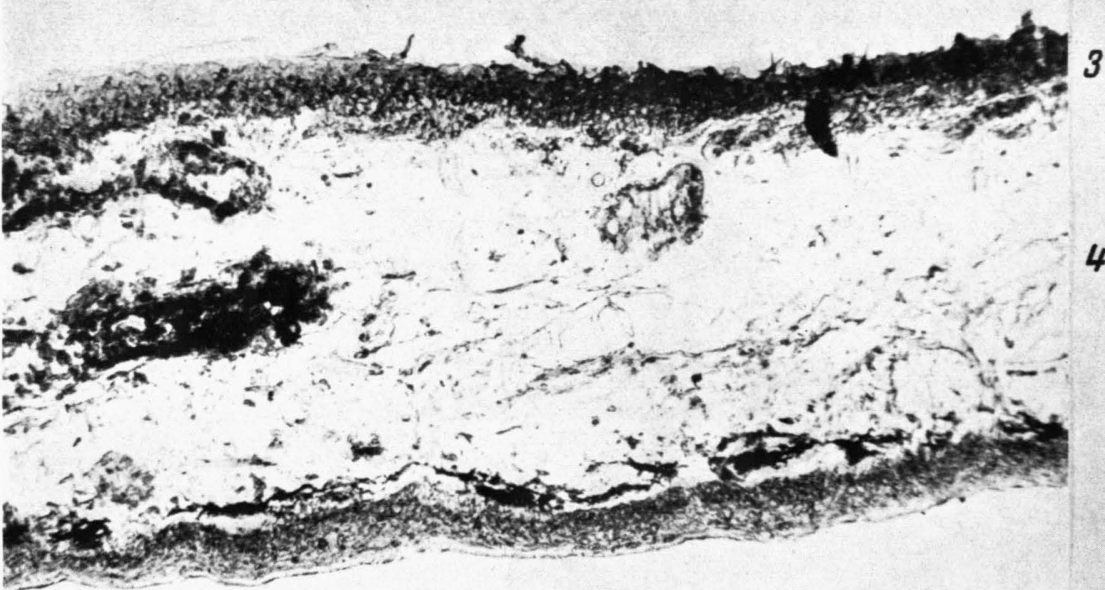

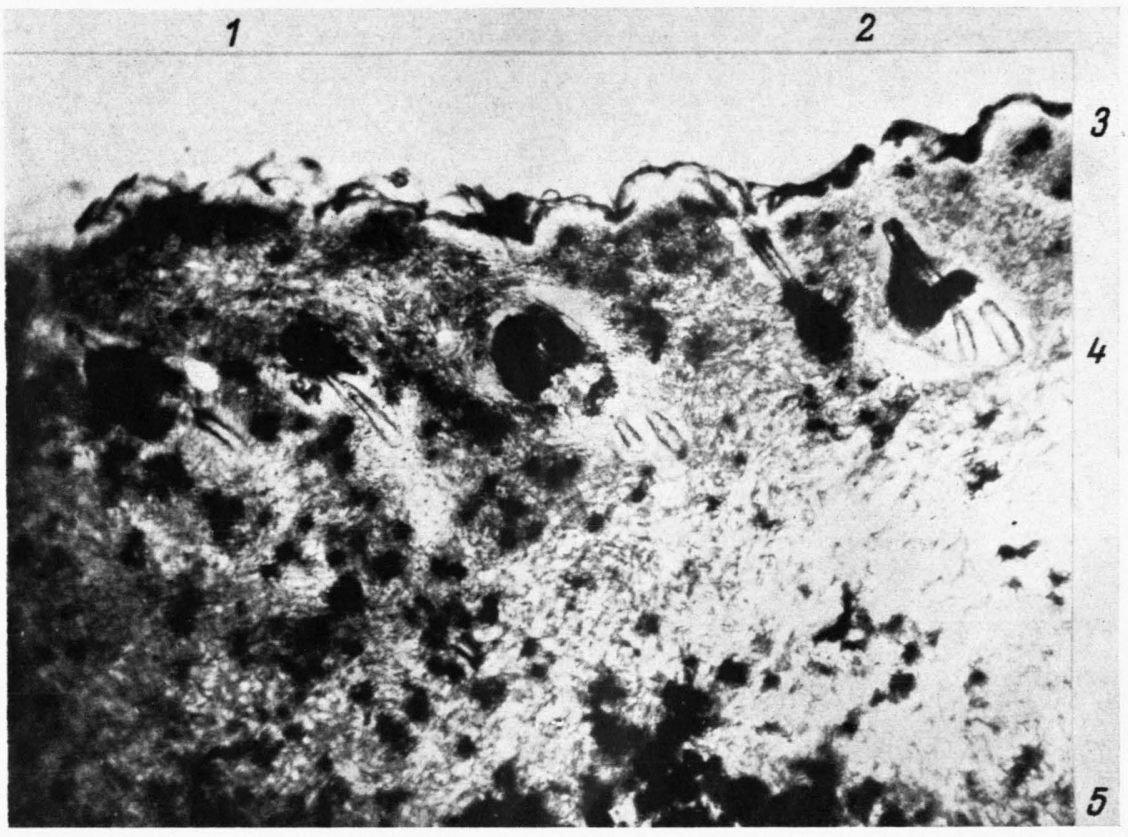

1

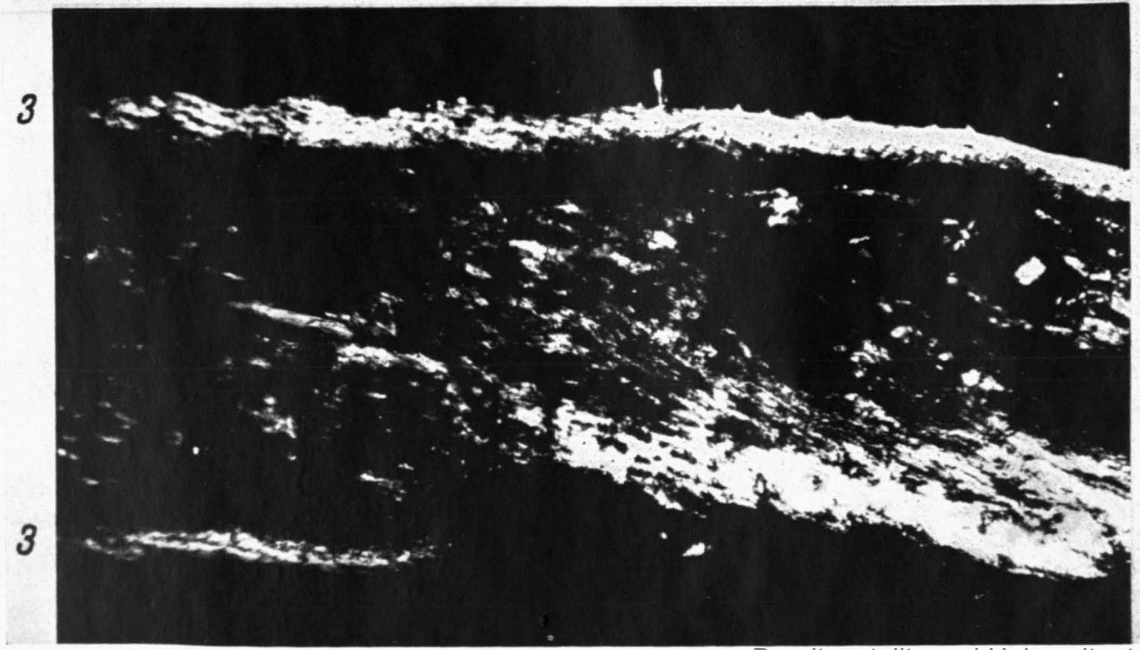

Abb. 4. Einwirkung von $\mathrm{N}$ a d i . Reagenz auf die bestrahlte Schwimmhaut bei $p_{\mathrm{H}} 4,4$ (Vergr. ca. 170-fach). Bestrahlt mit Hg.Br. 120 Min. 1 unbestr. Stelle, 2 bestr. Stelle, 3 Epidermis an der bestrahlten Stelle stark gebläut, 4 Corium ohne dicken Niederschlag.

Abb. 5. Rückenhaut der Maus nach Bestrahlung (Vergr. ca. 200-fach). 1 bestrahlte Stelle, 2 unbestr. Stelle, 3 Epidermis, 4 Corium mit IndophenolblauNiederschlag an der bestrahlten Stelle, 5 subcutanes Fettgewebe (Indophenolblau löst sich stark).

Abb. 6. Polarisationsoptische Aufnahme der bestrahlten Schwimmhaut (Vergr. ca. 170-fach). Strahleneinfall von unten. 1 unbestr. Stelle, 2 bestr. Stelle, 3 äußerste Epidermisschichten an der bestr. Stelle zerstört, 4 Corium, an der bestr. Stelle Fasern ausgerichtet. 